\title{
Relationship Between Psychological Overrun and Learning Burnout in Teenagers: Mediating Effect of Resilience
}

\author{
An Zhefeng, Du Beilei, Yang Fuge \\ Higher Education Research Institute of Faculty of Humanities and Social Sciences, Beijing University of Technology, Beijing, China
}

\section{Email address:}

anzf@bjut.edu.cn (An Zhefeng), 106441407@qq.com (Du Beilei), 1981065898@qq.com (Yang Fuge)

\section{To cite this article:}

An Zhefeng, Du Beilei, Yang Fuge. Relationship Between Psychological Overrun and Learning Burnout in Teenagers: Mediating Effect of Resilience. Science Innovation. Vol. 7, No. 2, 2019, pp. 81-85. doi: 10.11648/j.si.20190702.16

Received: March 1, 2019; Accepted: May 23, 2019; Published: June 15, 2019

\begin{abstract}
In order to examine the mediating effect of teenagers' resilience between psychological overrun and learning burnout, 305 teenagers were monitored on self-made psychological overrun scale, learning burnout scale and psychological resilience scale. Research results show: (1) significant positive correlation was found between psychological overrun and learning burnout among teenagers; significant positive correlation was found between psychological resilience and learning burnout among teenagers; the correlation between psychological resilience and learning burnout among teenagers was negative; (2) Resilience had a partial mediating effect on the relationship between psychological overrun and learning burnout among teenagers. This study validated the joint effects of teenagers' resilience, psychological overrun and learning burnout on the reduction of adolescents' burdens. It has a certain guiding significance for adolescents to improve their academic burdens and reduce learning burnout from a psychological perspective.
\end{abstract}

Keywords: Teenagers, Psychological Overrun, Learning Burnout, Resilience

\section{青少年心理超限与学习倦忩的关系: 心理弹性的中介效应}

\section{安哲锋, 杜蓓蕾, 杨馥歌 \\ 北京工业大学文法学部高等教育研究院, 北京, 中国}

\section{邮箱}

anzf@bjut.edu.cn（安哲锋）,106441407@qq.com（杜蓓蕾）,1981065898@qq.com（杨馥歌）

\begin{abstract}
摘要: 本研究采用自编的心理超限量表、学习倦急量表和心理弹性量表对305名青少年进行调查, 考察青少年心理超限 与学习倦怠的关系及心理弹性在其中的中介作用。研究结果表明：（1）青少年心理超限与学习倦怠正相关，与心理弹 性负相关; 青少年心理弹性与学习倦怠负相关。（2）青少年心理弹性在心理超限对学习倦急的影响中具有部分中介作 用。本研究验证青少年心理弹性、心理超限和学习倦急对青少年减负的联合作用, 对青少年从心理视角上改善学业负 担和减轻学习倦怠具有一定的指导意义。
\end{abstract}

关键词: 青少年, 心理超限, 学习倦急, 心理弹性 


\section{1. 引言}

青少年学业负担情况得到社会各界广泛的关注。其中, 过重的学业负担可能会导致青少年心理超限和学习倦急。 研究指出, 青少年负担过重会导致学生产生不自信、情感 枯竭和低成就感的现象 [1-3]。为了更好地了解青少年学业 负担情况, 探索其对青少年学习的影响机制, 本研究拟考 察青少年心理超限与学习倦急的关系以及心理弹性的中 介效应。

心理超限与学习倦怠都是青少年心理负担过重和学 习压力超载的不良后果, 两者都与心理弹性具有明显的相 关关系。具体而言, 心理弹性对心理超限具有负向预测作 用 [4], 心理弹性与学习倦急具有负相关的关系 [5-6]。研 究发现, 心理弹性通过保护性因素使中学生在学习逆境中 获得良好的发展, 不失为一种推动学习进程的有利因素 [7]。 研究指出, 心理弹性既对学习倦急产生直接影响, 也通过 积极情绪对学习倦怠产生间接影响 [8]。

然而, 现有研究多关注在心理超限与心理弹性或者心 理倦怠与心理弹性的相关性或三者各自的影响因素的研 究上, 较少探讨心理超限、学习倦急和心理弹性三者的关 系。截止到目前, 心理弹性还没有统一的定义, 对其概念 的研究主要分为结果性定义、品质性定义和过程性定义三 种[9]。综合以上三种定义, 本研究的心理弹性指的是青少 年的心理韧性、心理回复力 (心理恢复力) 、心理抗挫折 的能力, 是一种稳定的特征或心理品质, 有个体水平的高 低, 是随着身边环境的变化以及自我状态的变化, 进行自 我调适的过程。国内已经有许多研究者将心理弹性作为中 介因子进一步研究个体不同方面的内在关系, 心理弹性在 个体心理中所能起到的中介作用和调节作用已经得到充 分的证明[10-11]。然而对于青少年心理弹性在青少年心 理超限对学习倦急影响的中介作用目前还较少涉及, 本研 究将探讨青少年心理弹性在青少年心理超限与学习倦急 的中介作用。

\section{2. 方法}

\section{1. 被试}

施测对象取自北京市、辽宁省、吉林省和浙江省四个 地区的初中和高中，共选取初中和高中共 6 个年级被试 400 人并发放问卷 400 份，回收问卷 320 份，回收率达 $80 \%$, 无效问卷去除后剩余有效问卷 305 份, 有效率为 $95.31 \%$ 。 其中, 男生为 $43.6 \%$, 女生为 $56.4 \%$; 年级为高中占比 $46.6 \%$, 初中占比 $53.4 \%$; 就读所处地区北京市占比 $69.2 \%$, 非北 京市地区占比 $30.8 \%$ 。

\section{2. 研究工具}

\subsection{1. 自编心理超限量表}

根据对心理超限的定义的界定与文献的整理,并对其 结构进行维度上的划分, 通过对问题内容及其侧重点的反 复讨论与修改, 编制成由 20 个项目组成的“青少年心理超 限量表”。每个项目都由一句隐含心理超限行为的陈述句
组成, 下设 5 个备选答案, 让被试选择。采用Likert5级计 分制, 1 代表“极不符合”，5代表“非常符合”。数字越大, 表示心理超限程度越高。量表的内部一致性系数 $\alpha=$ 0.905( $\mathrm{n}=305)$ 。验证性因子分析显示, 剔除二个低于 0.5 的 题项，剩余所有题目的因子负荷在 0.706 和 0.893 之间。 因素分析的方差可解释率为 $80.368 \%$, 量表具有良好的结 构效度。

\subsection{2. 学习倦怠量表}

采用戴晓阳和吴艳 (2007) 编制的青少年学习倦急量 表, 共 16 个项目包括身心耗损、低成就感和学业疏离 3 个 维度。采用 Likert-5 点评分, 总分越高表示个体学习倦急 程度越高。本研究中该量表的 $\alpha$ 系数为 0.855 。

\subsection{3. 心理弹性量表}

胡月琴和甘怡群 (2008) 编制的青少年心理韧性量表 对本研究中的被试的心理弹性进行测量, 该量表要求被试 对 27 个项目做出 $1 \sim 5$ 五点评分, 分值越高, 表明个体心理 弹性适应性越高。本研究中该量表的 $\alpha$ 系数为 0.879 。

\section{3. 程序}

问卷填写采用无记名方式，被试完成整个测试大约 需要 25 分钟。使用SPSS19.0进行数据分析处理。由于需 要验证心理弹性在心理超限与学习倦急的关系中的中介 作用, 因此采用AMOS23.0中使用Bootstrap方法运行 5000 次。

\section{3. 结果}

\section{1. 各变量的相关}

相关分析表明, 心理超限与学习倦急呈显著正相关, 与心理弹性呈显著负相关; 心理弹性与学习倦急呈显著负 相关（如表1）。

表1 各变量的相关。

\begin{tabular}{llllll}
\hline & $\mathbf{M}$ & SD & $\mathbf{1}$ & $\mathbf{2}$ & $\mathbf{3}$ \\
\hline 1 心理超限 & 2.649 & 0.858 & 1 & & \\
2 心理弹性 & 3.442 & 0.562 & $-.453^{* *}$ & 1 & \\
3 学习倦急 & 2.707 & 0.665 & $.703^{* *}$ & $-.557^{* *}$ & 1 \\
\hline
\end{tabular}

(注: $\mathrm{M}$ 为平均数, $\mathrm{SD}$ 为标准差; ${ }^{*}, \mathrm{p}<0.05 ;{ }^{* *}, \mathrm{p}<0.01 ; * * *, \mathrm{p}<0.001$,下同)

\section{2. 总效应、中介效应及直接效应}

本研究采用AMOS23.0中使用Bootstrap方法来分别检 验整体样本、男生样本、女生样本、高中生样本和初中生 样本, 青少年心理弹性在心理超限与学习倦急关系的总效 应和中介效应的显著性。从表2整体样本可以看到, 心理 超限对学习倦急的总效应值为 0.704 , Bias-Corrected 与 Percentile $95 \%$ CI的Lower和Upper的值区间之内均不包含 0 , 表明总效应均存在; 心理超限通过心理弹性对学习倦急的 中介效应值为 0.136 , 占比为 $0.136 / 0.704=19.3 \%$, 在 Bias-Corrected 与Percentile $95 \%$ CI 的Lower和Upper的值 
区间之内均不包含 $0 ，$ 表明中介效应均存在; 心理超限对 学习倦急的直接效应值为 0.568 , 在 Bias-Corrected 与 Percentile $95 \%$ CI的Lower和Upper的值区间之内均不包含 0 , 表明直接效应均存在。对男生样本、女生样本、高中生样
本和初中样本总效应和中介效应的显著性分析详见表3、4、 5 和6。综上可以得到心理弹性在整体样本、不同性别和各 个学段都在心理超限对学习倦急的影响中具有部分中介 作用。

表2 整体样本总效应、中介效应及直接效应的Bootstrap检验（5000次抽样）。

\begin{tabular}{|c|c|c|c|c|c|}
\hline & \multirow{3}{*}{ 标准化效应值 } & \multirow{2}{*}{\multicolumn{2}{|c|}{$\begin{array}{l}\text { Bias-Corrected } \\
95 \% \mathrm{CI}\end{array}$}} & \multicolumn{2}{|c|}{ Percentile } \\
\hline & & & & $95 \% \mathrm{CI}$ & \\
\hline & & Lower & Upper & Lower & Upper \\
\hline \multicolumn{6}{|l|}{ 总效应 } \\
\hline $\begin{array}{l}\text { 心理超限-学习倦急 } \\
\text { 间接效应 }\end{array}$ & 0.704 & 0.635 & 0.761 & 0.638 & 0.762 \\
\hline $\begin{array}{l}\text { 心理超限-心理弹性-学习倦急 } \\
\text { 直接效应 }\end{array}$ & 0.136 & 0.088 & 0.194 & 0.085 & 0.19 \\
\hline 心理超限-学习倦怠 & 0.568 & 0.48 & 0.648 & 0.483 & 0.65 \\
\hline
\end{tabular}

表3 男生样本总效应、中介效应及直接效应的Bootstrap检验（5000次抽样）。

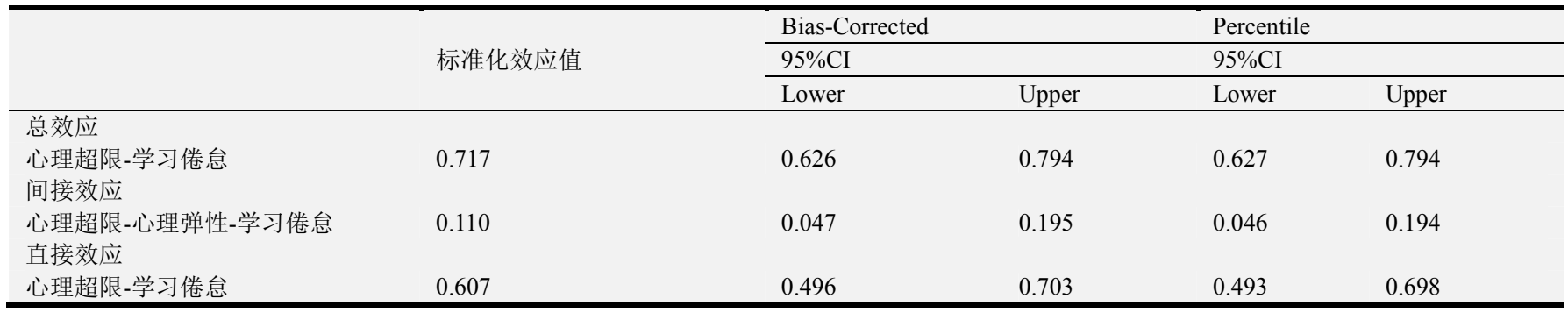

表4 女生总效应、中介效应及直接效应的Bootstrap检验（5000次抽样）。

\begin{tabular}{|c|c|c|c|c|c|}
\hline & \multirow{3}{*}{ 标准化效应值 } & \multicolumn{2}{|l|}{ Bias-Corrected } & \multicolumn{2}{|c|}{ Percentile } \\
\hline & & $95 \% \mathrm{CI}$ & & $95 \%$ CI & \\
\hline & & Lower & Upper & Lower & Upper \\
\hline \multicolumn{6}{|l|}{ 总效应 } \\
\hline $\begin{array}{l}\text { 心理超限-学习倦急 } \\
\text { 间接效应 }\end{array}$ & 0.693 & 0.591 & 0.773 & 0.597 & 0.777 \\
\hline $\begin{array}{l}\text { 心理超限-心理弹性-学习倦急 } \\
\text { 直接效应 }\end{array}$ & 0.157 & 0.087 & 0.246 & 0.071 & 0.231 \\
\hline 心理超限-学习倦怠 & 0.536 & 0.410 & 0.665 & 0.420 & 0.675 \\
\hline
\end{tabular}

表5 高中生总效应、中介效应及直接效应的Bootstrap检验（5000次抽样）。

\begin{tabular}{|c|c|c|c|c|c|}
\hline & \multirow{3}{*}{ 标准化效应值 } & \multicolumn{2}{|l|}{ Bias-Corrected } & \multicolumn{2}{|c|}{ Percentile } \\
\hline & & $95 \% \mathrm{CI}$ & & $95 \% \mathrm{CI}$ & \\
\hline & & Lower & Upper & Lower & Upper \\
\hline \multicolumn{6}{|l|}{ 总效应 } \\
\hline $\begin{array}{l}\text { 心理超限-学习倦急 } \\
\text { 间接效应 }\end{array}$ & 0.696 & 0.595 & 0.778 & 0.600 & 0.781 \\
\hline $\begin{array}{l}\text { 心理超限-心理弹性-学习倦怠 } \\
\text { 直接效应 }\end{array}$ & 0.105 & 0.024 & 0.212 & 0.023 & 0.210 \\
\hline 心理超限-学习倦怠 & 0.590 & 0.449 & 0.693 & 0.445 & 0.696 \\
\hline
\end{tabular}

表6 初中生总效应、中介效应及直接效应的Bootstrap检验（5000次抽样）。

\begin{tabular}{|c|c|c|c|c|c|}
\hline & \multirow{3}{*}{ 标准化效应值 } & \multicolumn{2}{|l|}{ Bias-Corrected } & \multicolumn{2}{|c|}{$\overline{\text { Percentile }}$} \\
\hline & & $95 \% \mathrm{CI}$ & & $95 \%$ CI & \\
\hline & & Lower & Upper & Lower & Upper \\
\hline \multicolumn{6}{|l|}{ 总效应 } \\
\hline 心理超限-学习倦怠 & 0.705 & 0.604 & 0.785 & 0.610 & 0.790 \\
\hline 间接效应 & & & & & \\
\hline $\begin{array}{l}\text { 心理超限-心理弹性-学习倦急 } \\
\text { 直接效应 }\end{array}$ & 0.157 & 0.100 & 0.232 & 0.091 & 0.221 \\
\hline 心理超限-学习倦怠 & 0.549 & 0.447 & 0.654 & 0.451 & 0.658 \\
\hline
\end{tabular}




\section{4. 讨论}

\section{1. 心理超限、心理弹性和学习倦急的相关分析}

心理超限与学习倦急进行相关分析, 得到各个变量均 与学习倦怠呈显著的正相关。青少年的心理超限得分越 低, 学习倦急的得分越低; 心理超限得分越高, 学习倦急 的得分越高。心理超限是人的心理内部情绪和思想, 学 习倦急是个体学习疲累并伴随言行举止的变化。当一个 青少年进入心理超限状态时, 他/她对学习和身边的事物 已经持有消极的态度, 此时, 这位青少年面对学习压力就 会产生学习倦急, 采用课堂隐性逃学等方式。

心理超限与心理弹性进行相关分析, 数据表明, 各个 变量均与心理弹性呈显著的负相关。心理弹性分数越高, 心理超限分数越低, 心理弹性对心理超限具有负向预测作 用。当青少年心理弹性水平较高时, 青少年的情绪起伏会 趋于平缓, 看待事物的心理视角相对客观, 心理状态也会 比较稳定, 不易发生心理超限的情况。

心理弹性与学习倦急进行相关分析得到, 各变量均与 学习倦怠均具有显著的负相关。心理弹性分数越高, 学习 倦急分数越低, 心理弹性对学习倦怠具有负向预测作用。 心理弹性水平较低的青少年, 面临学习困境时, 可能会采 取相对消极的方式和持有消极的态度, 比如面临学习难题, 他/她可能就会退缩或不能坚定地完成学习目标, 进而放 弃自我, 造成学习倦怠。

心理超限、心理弹性、学习倦急进行相关分析得到, 三者之间均具有显著的相关关系。说明三者之间有着紧密 的相关关系, 通过判定心理弹性的水平, 可以间接推断出 心理超限和学习倦怠的发展现状。

因此, 为确保青少年的心理水平处在一个安全且合 理的区间内, 及时预防或解决青少年心理超限状态的出现, 建立青少年心理信息反馈渠道, 可以采取一些辅助措施。 例如老师可以定期做心理调查 (电子) 问卷, 控制题目数 量, 避免重复, 在提交答案后, 可以设置简短的有趣的建 议, 在了解青少年心理状态的同时不带给其困扰, 寓教于 无形之中。根据反馈得来的信息建立解决渠道, 制定长期 解决目标, 循序渐进, 做到隐性消除青少年心理超限和学 习倦怠。

\section{2. 青少年心理弹性中介效应}

青少年的心理弹性在心理超限对学习倦怠的影响中 具有部分中介作用, 这说明通过心理弹性可以同时直接作 用于心理超限和学习倦怠, 而心理超限与学习倦急可以通 过心理弹性间接作用于对方。当青少年同时出现心理超限 和学习倦急时, 他/她的心理弹性一定处于一个较低的水 平, 当他/她拥有较高水平的心理弹性时, 出现心理超限 和学习倦怠的概率也会随之降低。

青少年中男生的心理弹性在心理超限对学习倦急的 影响中具有部分中介作用; 青少年中女生的心理弹性在心 理超限对学习倦急的影响中具有部分中介作用。高中生的 心理弹性在心理超限对学习倦急的影响中具有部分中介 作用; 初中生的心理弹性在心理超限对学习倦急的影响中
具有部分中介作用。由此可见, 心理弹性在不同的性别和 学段均发挥部分中介作用, 心理弹性是每个阶段每个青少 年都必备的, 青少年通过心理弹性调节自己的心理活动, 各个心理活动之间通过心理弹性互相影响, 促进或制约对 方的成长, 同时, 心理弹性也是心理活动的动力, 青少年 不仅通过心理弹性促进或削弱其他心理活动, 也会通过外 在的行为与内在的心理活动对心理弹性的弹力成长起到 积极或消极的影响。

青少年的心理超限可以直接作用于学习倦怠, 又可以 通过心理弹性的中介效应作用于学习倦急, 起到部分中介 作用。心理超限和学习倦怠作为青少年个体消极状态的两 种表达方式, 都与青少年的主观意愿紧密相连, 通过主观 的心理弹性将二者联系到一起, 可以更加直观地发现青少 年个体的动态变化, 这可以对青少年的成长贡献一定的力 量, 帮助他们及时地调整, 培养更加积极更加符合社会发 展与个体发展的青少年。

\section{3. 研究局限和实践意义}

本研究仍然存在一些局限:（1）由于采取随机发放 问卷的方式, 问卷样本有限, 样本的典型性有待商榷; (2) 本研究只采用了问卷调查法, 存在调查方法有限的问题, 同时问卷设置题目较多, 存在青少年填写问卷不认真或是 填写中途放弃等问题。

本研究结果对教育实践有重要启示。针对心理弹性在 心理超限与学习倦急的关系中所扮演的角色, 以心理弹性 为中心点, 将心理超限与学习倦急视为环绕在其身边的圆 环, 利用心理弹性的中介作用, 将三者的互动关系充分运 用到实际中。

首先, 老师与家长通过对青少年的学习状态的观察与 评估, 如果当青少年的学习行为明显出现对学习的厌烦, 老师与家长应当对青少年的其他生活行为进行追踪观察, 确认出现学习倦急后, 可以通过沟通交流的方式探寻其是 否出现心理超限, 进一步判定其心理弹性的水平。

其次, 确认青少年状态后, 通过增强心理弹性和预防 心理超限对学习倦怠进行及时的帮助与引导, 防止情况恶 化。

最后, 将青少年心理超限与学习倦急的出现状态及产 生原因、超限状态的反应时长、解决效果等内容整理, 并 建立匿名档案, 为以后相似案例提供帮助, 同时, 不仅实 现了心理超限的实际应用, 还可以将本研究的量化研究延 伸到质性研究, 丰富心理超限的研究体系与研究内容, 做 到不仅有预防机制, 还有解决的方案。

\section{5. 结论}

数据统计分析表明本研究所取青少年样本的心理超 限水平较低, 心理弹性水平较高, 学习倦急水平较低。通 过相关分析、回归分析和中介检验等证明在青少年心理超 限与学习倦怠的关系中, 心理弹性确实存在间接的中介作 用。

部分青少年存在心理超限和学业超负的情况, 但不是 普遍存在; 青少年的心理超限与学习倦怠也确实存在一种 
动态的相关关系, 两者都与心理弹性存在负相关的关系, 即一个个体的心理出现超限, 那么他/她的学习倦急可能 也已经出现; 由于心理弹性对心理超限和学习倦急都具有 负向的预测和调控功能, 当我们通过观察和其它方式发现 青少年出现心理超限或学习倦急时, 可以通过外部帮助增 强其心理弹性的方式对两者进行减轻与缓解，从而使青少 年更好地学习与成长。

\section{参考文献}

[1] 杨丽䢞,连榕.学习倦急的研究现状及展望 [J].集美大学学报 (教育科学版),2005(02):54-58。

[2] 间旭蕾.教育中的“肉”与“灵”——身体社会学研究[M].南京: 南京师范大学出版社, 2007:4。

[3] 胡俏, 戴春林. 中学生学习倦急结构研究 [J]. 心理科 学,2007(01):162-164+195。

[4] 孟子明.大学生压力、心理弹性与心理安全感的关系研究[D]. 广西师范学院,2017。
[5] 陈慧. 高三学生心理弹性与学习倦急的关系研究[D].南昌大 学,2014。

[6] 李媛.初中生感知学校气氛、心理弹性与学习倦急的关系研 究[D].河北师范大学,2016。

[7] 阮微.心理弹性对中学生学习倦急的积极影响[J]. 中国科教 创新导刊, 2012(15):223-223。

[8] 张卓尔.中学生心理弹性对学习倦急的影响[D].湖南科技大 学,2016。

[9] 邓多林.初中生生活事件、心理弹性与生活满意度的关系研 究[D].湖南师范大学,2013。

[10] 韦耀阳.大学生成就动机与焦虑的关系:心理弹性的中介作 用 $[\mathrm{J}]$. 湖北理工学院学报 (人文社会科学 版),2017,34(05):83-88。

[11] 王雅倩,王丽,向光富.大学生大五人格与主观幸福感:心理弹 性的中介作用[J].中国健康心理学杂志,2017,25(01):112-116。 